\title{
Ultra High Energy Cosmic Rays from Engine-driven Relativistic Supernovae
}

\author{
Sayan Chakraborti \\ Tata Institute of Fundamental Research, \\ 1 Homi Bhabha Road, Mumbai, India \\ email: sayan@tifr.res.in
}

\begin{abstract}
The sources of the highest energy cosmic rays remain an enigma half a century after their discovery. Understanding their origin is a crucial step in probing new physics at energies unattainable by terrestrial accelerators. They must be accelerated in the local universe as otherwise interaction with cosmic background radiations would severely deplete the flux of protons and nuclei at energies above the Greisen-Zatsepin-Kuzmin (GZK) limit. Hypernovae, nearby GRBs, AGNs and their flares have all been suggested and debated in the literature as possible sources. Type Ibc supernovae have a local sub-population with mildly relativistic ejecta which are known to be sub-energetic GRBs or X-Ray Flashes for sometime and more recently as those with radio afterglows but without detected GRB counterparts, such as SN 2009bb. In this talk we present the size-magnetic field evolution, baryon loading and energetics of SN 2009bb using its radio spectra obtained with VLA and GMRT. We show that the engine-driven SNe lie above the Hillas line and they can explain the characteristics of post-GZK UHECRs.
\end{abstract}

Keywords. cosmic rays, supernovae: individual (SN 2009bb, SN 2012ap), gamma rays: bursts

\section{Introduction}

The Pierre Auger Collaboration (2007) show that the ultra high energy cosmic rays (UHECRs) have such a large energy and a low flux. They were first detected by Linsley (1963) only by air showers where according to Bhabha \& Heitler (1937) the Earth's atmosphere acts as the active medium. UHECRs beyond the GZK limit have been invoked by Coleman, S. \& Glashow (1999) to propose tests of known physical laws and symmetries. Understanding their origin is important for their use as probes of new physics. The magnetic rigidity of these particles are such that the magnetic fields in our galaxy are neither strong enough to contain them nor bend them sufficiently. However UHECR protons at energies above $60 \mathrm{EeV}$ can interact with Cosmic Microwave Background (CMB) photons via the $\Delta$ resonance. The cross section of this process is such that only local extragalactic cosmic ray sources within $200 \mathrm{Mpc}$ of the Earth can contribute significantly to the flux of UHECRs above the GZK limit found by Greisen (1966), Zatsepin \& Kuz'min (1966), The High Resolution Fly'S Eye Collaboration (2008). Since particles of such high energy could not have traveled to Earth from cosmological distances, unless Lorentz invariance breaks down at these energies, as proposed by Coleman, S. \& Glashow (1999), their detection encourages the search for potential cosmic ray accelerators in the local Universe. Accordingly Waxman (1995), Milgrom \& Usov (1995), Murase et al. (2006) suggested nearby GRBs, Wang et al. (2007), Budnik et al. (2008) Hypernovae, The Pierre Auger Collaboration (2007) AGNs and Farrar \& Gruzinov (2009) their flares, as possible sources.

SNe with relativistic ejecta have been detected until recently, exclusively through associated Long GRBs like GRB 980425 or its twin GRB 031203. Soderberg et al. (2006) 


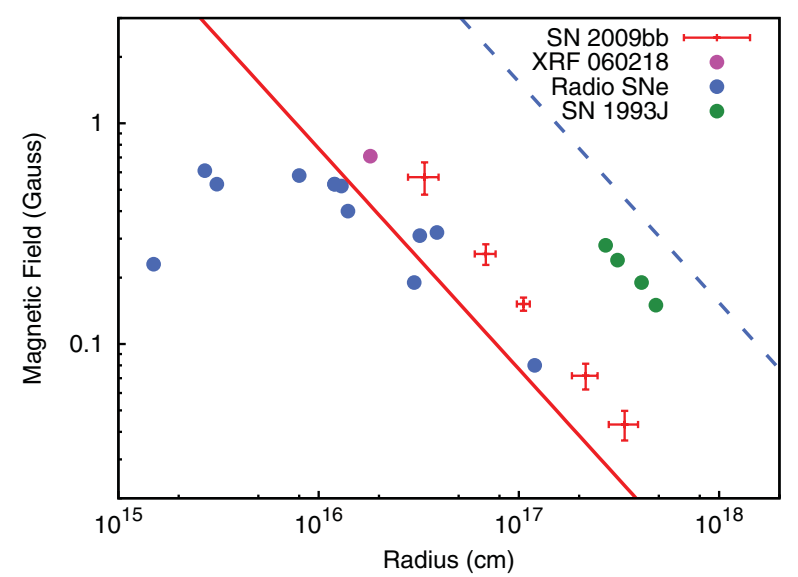

Figure 1. Hillas Diagram: Mildly relativistic sources $(\beta / \Gamma \sim 1)$ must lie above the solid line, to accelerate Iron nuclei to $60 \mathrm{EeV}$ by diffusive shock acceleration, according to $E_{Z} \lesssim \beta e Z B R / \Gamma$. Non-relativistic $\mathrm{SNe}(\beta / \Gamma \sim 0.05)$ must lie above the dashed line to reach the same energies. Radius and magnetic field of SN 2009bb (crosses, at 5 epochs, determined here from radio observations with VLA and GMRT assuming equipartition) and XRF 060218 lie above the solid red line. Other balls denote other radio SNe from Chevalier (1998). For SN 1993J only, the magnetic fields are obtained by Chandra, Ray, \& Bhatnagar (2004) without assuming equipartition. All non-relativistic SNe including SN 1993J lie below the dashed line and are unable to produce UHECRs unlike the mildly relativistic SN 2009bb and XRF 060218 which lie above the solid line.

showed that mildly relativistic SNe, like SN 2006aj associated with XRF 060218, are hundred times less energetic but thousand times more common (in their isotropic equivalent rate, the relevant rate for UHECRs reaching the observer) than classical GRBs. Soderberg et al. (2010) now discovered the presence of a radio emitting engine driven outflow from SN 2009bb, without a detected GRB. These mildly relativistic SNe, a subset of SNe Ibc detected either using X-Ray Flashes (XRFs) or radio afterglows, are far more abundant at low redshifts required for the UHECR sources, than the classical GRBs. Their mildly relativistic nature makes them have the most favorable combination of $\beta / \Gamma \sim 1$, unlike both non-relativistic SNe and ultra-relativistic classical Long GRBs. In Chakraborti et al. (2010) we measured the size and magnetic field of the prototypical SN 2009bb at several epochs. Such engine-driven supernovae are placed above the Hillas line and we demonstrate that they may accelerate cosmic rays beyond the GZK threshold. Together with the rates and energetics of such events, we establish that they readily explain the post-GZK UHECRs.

\section{Magnetic field evolution with expanding radius}

In order to derive the highest energy upto which these relativistic SNe can accelerate cosmic rays, we determine the evolution of the size and the magnetic field in the blastwave. Radii and magnetic fields were measured from VLA and GMRT data at 5 epochs, for plotting on the Hillas diagram (Figure 1).

With a set of assumptions for the electron energy distribution and magnetic fields the radius of the forward shock wave at the time of the synchrotron self-absorption peak was given by Chevalier (1998) as

$$
R \bumpeq 4.0 \times 10^{14} \alpha^{-1 / 19}\left(\frac{f}{0.5}\right)^{-1 / 19}\left(\frac{F_{o p}}{\mathrm{mJy}}\right)^{9 / 19}\left(\frac{D}{\mathrm{Mpc}}\right)^{18 / 19}\left(\frac{\nu}{5 \mathrm{GHz}}\right)^{-1} \mathrm{~cm},
$$


where $\alpha=\epsilon_{e} / \epsilon_{B}$ is the ratio of relativistic electron energy density to magnetic energy density, $f$ is the fraction of the spherical volume occupied by the radio emitting region, $F_{o p}$ is the observed peak flux, and $D$ is the distance. Using the same variables, the magnetic field is given by

$$
B \simeq 1.1 \alpha^{-4 / 19}\left(\frac{f}{0.5}\right)^{-4 / 19}\left(\frac{F_{o p}}{\mathrm{mJy}}\right)^{-2 / 19}\left(\frac{D}{\mathrm{Mpc}}\right)^{-4 / 19}\left(\frac{\nu}{5 \mathrm{GHz}}\right) \mathrm{G} .
$$

The radio spectrum of SN 2009bb at all epochs from discovery, paper by Soderberg et al. (2010) and Chakraborti et al. (2010), as obtained from observations using the Very Large Array (VLA) and the Giant Metrewave Radio Telescope (GMRT), is well fit by the SSA model. Thus we can measure the size and magnetic field of a candidate accelerator, instead of indirect arguments connecting luminosity with the Poynting flux.

SN 2009bb and XRF 060218 can both confine UHECRs and accelerate them to the highest energies seen experimentally. At the time of the earliest radio observations (Soderberg et al. (2010)) the combination of $\beta / \Gamma \sim 1$ for SN $2009 \mathrm{bb}$ shows that it could have accelerated nuclei of atomic number $Z$ to an energy of $\sim 6.5 \times Z \mathrm{EeV}$. Thus the source could have accelerated protons, Neon, and Iron nuclei to 6.4, 64 and $166 \mathrm{EeV}$, respectively. Here the highest energy particles are likely to be nuclei heavier than protons, consistent with the latest results from The Pierre Auger Collaboration (2010) indicating an increasing average rest mass of primary UHECRs with energy. Therefore, our results support the Auger collaboration's claimed preference of heavier UHECRs at the highest energies.

\section{Rates of Engine-driven Supernovae}

We require the rate of relativistic SNe to estimate whether there are enough of them to explain the target objects associated with the $\sim 60$ detected UHECRs. SNe Ibc occur at a rate of $\sim 1.7 \times 10^{4} \mathrm{Gpc}^{-3} \mathrm{yr}^{-1}$. The fraction that have relativistic outflows is still somewhat uncertain, estimated to be around $\sim 0.7 \%$ (Soderberg et al. (2010)). Hence the rate of SN 2009bb-like mildly relativistic SNe is $\sim 1.2 \times 10^{-7} \mathrm{Mpc}^{-3} \mathrm{yr}^{-1}$, comparable to the rate of mildly relativistic SNe detected as sub-energetic GRBs or XRFs of $\sim 2.3 \times 10^{-7} \mathrm{Mpc}^{-3} \mathrm{yr}^{-1}$. This leads to $\sim 4$ (or 0.5 ) such objects within a distance of 200 (or 100) Mpc every year. Since SN 2009bb is still a unique object, only a systematic radio survey can establish their cosmic rate and statistical properties. However, cosmic rays of different energies have different travel delays due to deflections by magnetic fields. For a conservative mean delay (Farrar \& Gruzinov $(2009)$ ) of $\left\langle\tau_{\text {delay }}\right\rangle \approx 10^{5}$ yrs we may receive cosmic rays from any of 4 (or 0.5$) \times 10^{5}$ possible sources at any given time. Since a direct association between a detected UHECR and its source is unlikely (Kashti \& Waxman (2008)), most workers have focused on the constraints placed on plausible sources (Hillas (1984), Waxman \& Loeb (2009)). Our arguments above show that this new class of objects satisfy all such constraints.

\section{Energy Injection and Energy Budget}

The required energy injection rate per logarithmic interval in UHECRs is $\Gamma_{i n j}=$

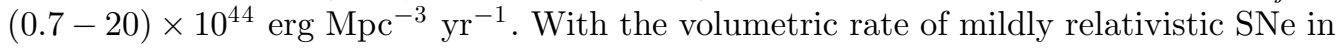
the local universe, if all UHECR energy injection is provided by local mildly relativistic $\mathrm{SNe}$, then each of them has to put in around $E_{S N}=(0.3-9) \times 10^{51}$ ergs. This is comparable to the kinetic energy in even a normal SN and can easily be supplied by a collapsar model. The mildly relativistic outflow of SN 2009bb is in nearly free expansion 
for $\sim 1$ year. Our measurements of this expansion show that this relativistic outflow, without a detected GRB, is significantly baryon loaded and the energy carried by the relativistic baryons is $E_{\text {Baryons }} \gtrsim 3.3 \times 10^{51}$ ergs (Chakraborti et al. (2010)).

\section{Conclusion}

We have shown that the newly found subset of nearby SNe Ibc, with engine-driven mildly relativistic outflows detected as sub-energetic GRBs, XRFs or solely via their strong radio emission, can be a source of UHECRs with energies beyond the GZK limit. Our study shows, for the first time, a new class of objects, which satisfy the constraints which any proposed accelerator of UHECRs has to satisfy. The author thanks Alicia Soderberg, Alak Ray, Abraham Loeb and Poonam Chandra for collaboration.

\section{References}

Bhabha, H. J. \& Heitler, W. 1937, Royal Society of London Proceedings Series A, 159, 432

Budnik, R., Katz, B., MacFadyen, A., \& Waxman, E. 2008, ApJ, 673, 928

Chakraborti, S., et al. 2010, Nature Communications, 2, 175

Chandra, P., Ray, A., \& Bhatnagar, S. 2004, ApJ, 612, 974

Chevalier, R. A. $1998, A p J, 499,810$

Coleman, S. \& Glashow, S. L. 1999, Phys. Rev. D, 59, 116008

Farrar, G. R. \& Gruzinov, A. 2009, ApJ, 693, 329

Greisen, K. 1966, Phys. Rev. Lett., 16, 748

Hillas, A. M. 1966, ARAA, 22, 425

Horiuchi, S., Murase, K., Ioka, K., \& Meszaros, P. 2012, ArXiv e-prints, astro-ph.HE:1203.0296

Kashti, T. \& Waxman, E. 2008, Journal of Cosmology and Astro-Particle Physics, 5, 6

Linsley, J. 1963, Phys. Rev. Lett., 10, 146

Milgrom, M. \& Usov, V. 1995, Ap. Lett., 449, 37

Murase, K., Ioka, K., Nagataki, S., \& Nakamura, T. 2006, Ap. Lett., 651, 5

Soderberg, A. M., Kulkarni, S. R., Nakar, E., et al. 2006, Nature, 442, 1014

Soderberg, A. M., Chakraborti, S., Pignata, G., et al. 2010, Nature, 463, 513

The High Resolution Fly'S Eye Collaboration 2008, Phys. Rev. Lett., 100, 101101

The Pierre Auger Collaboration 2007, Science, 318, 938

The Pierre Auger Collaboration 2010, Phys. Rev. Lett., 104, 091101

Wang, X.-Y., Razzaque, S., Mészáros, P., \& Dai, Z.-G. 2007, Phys. Rev. D, 76, 083009

Waxman, E. 1995, Phys. Rev. Lett., 75, 386

Waxman, E. \& Loeb, A. 2009, Journal of Cosmology and Astro-Particle Physics, 8, 26

Zatsepin, G. T. \& Kuz'min, V. A. 1966, Soviet Journal of Experimental and Theoretical Physics Letters, 4, 78

\section{Discussion}

Metzer: Is this proposal consistent with the Auger observations of the composition of UHECRs? Chakraborti: The depth of maxima measurements from The Pierre Auger Collaboration (2007) favour a high atomic weight for their highest energy showers. This is consistent with our picture as we favour high atomic numbers such as Iron.

IOKA: If the Cosmic Rays are accelerated at the forward shock, the iron fraction is not high. So the Injection efficiency would have to be higher for iron than protons. Chakraborti: It is possible that the relativistic outflow which originated from near the core of the progenitor may carry some nucleosynthetic products. Whether such nuclei can survive in jets is being investigated by Horiuchi et al. (2012). 